\title{
Influence of Electrostatic and Magnetic Fields on Hot Electron Emission in Ultra-Intense Laser Matter Interactions
}

\author{
Toshinori YABUUCHI ${ }^{1,2)}$, Yasuhiko SENTOKU ${ }^{3)}$, Takeshi MATSUOKA ${ }^{2)}$, Hideaki HABARA ${ }^{1,2)}$, \\ Ken ADUMI ${ }^{1,2)}$, Zenglin $\mathrm{CHEN}^{2)}$, Ryosuke KODAMA ${ }^{1,2)}$, Kiminori KONDO ${ }^{1,2)}$, Anle $\mathrm{LEI}^{2)}$, \\ Kunioki MIMA ${ }^{2)}$, Motonobu TAMPO ${ }^{2)}$, Tsuyoshi TANIMOTO ${ }^{1,2)}$ and Kazuo A. TANAKA ${ }^{1,2)}$ \\ ${ }^{1)}$ Graduate School of Engineering, Osaka University, Osaka 565-0871, Japan \\ ${ }^{2)}$ Institute of Laser Engineering, Osaka University, Osaka 565-0871, Japan \\ ${ }^{3)}$ Department of Physics, University of Nevada, Reno, Nevada 89557, USA
}

(Received 15 February 2007 / Accepted 2 April 2007)

\begin{abstract}
We studied the influence of electrostatic and magnetic fields at the rear surface of solid targets on the hot electron emission generated by ultra-intense laser pulses. The number of emitted electrons increases in experiments when a pre-plasma is created on the target rear surface. The formation of the electrostatic potential is clearly retarded in particle-in-cell simulations resulting in the elongation of time window for hot electron emission. The increase in the number of electrons is consistent with the Alfvén current within the elongated time window.
\end{abstract}

(c) 2007 The Japan Society of Plasma Science and Nuclear Fusion Research

Keywords: ultra-intense laser, hot electron, electrostatic potential, retarded potential, Alfvén current

DOI: $10.1585 /$ pfr.2.015

Hot electrons generated by an ultra-intense laser (UIL) pulse are regarded as an energy carrier for the core heating in fast ignition laser fusion scheme [1]. Up to $40 \%$ energy conversion efficiency is observed from UIL to hot electrons with the $K_{\alpha}$ fluorescence technique inside a solid target [2]. However, it is known that the total energy of hot electrons observed in vacuum is $1 \%$ or less of the laser energy [3]. This discrepancy may be closely related to the self-generated strong electrostatic and magnetic fields produced at the target rear side.

We have studied the influence of the fields on the hot electron emission creating a pre-plasma on the target rear surface (rear plasma). The rear plasma effect is preliminarily studied for the electrostatic field production on the ion acceleration [4]. We measure the total number of electrons emitted through the target irradiated by the UIL pulse. The observed number of electrons increases when the rear plasma is created. The increase may be closely related to the electrostatic field or the potential evolution affected by the rear plasma. The potential evolution is studied with a one dimensional (1D) particle-in-cell (PIC) simulation in two cases: with and without rear plasma. The influence of self-excited magnetic field is also discussed based on the Alfvén limit [5].

The experiments are carried out using a $30 \mathrm{TW}$ laser facility, Gekko Module II at the Institute of Laser Engineering (ILE), Osaka University. The energy and time duration of the UIL pulse are 2-10 J and $700 \mathrm{fs}$, respectively. The UIL pulse interacts with a $50-\mu \mathrm{m}$-thick aluminum foil target at a maximum intensity $3 \times 10^{18} \mathrm{~W} / \mathrm{cm}^{2}$

author'se-mail: tyabu@ile.osaka-u.ac.jp

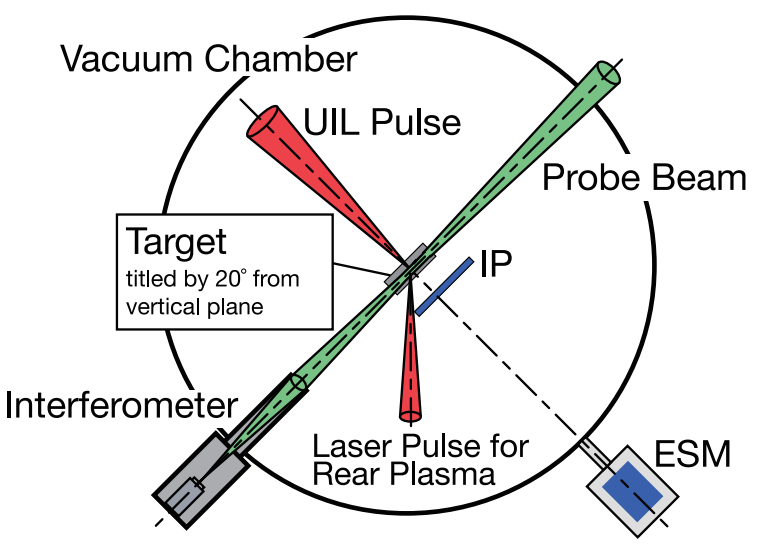

Fig. 1 Top view of the experimental setup.

in a $s$-polarization with an incidence angle of $20^{\circ}$. Separated laser pulse irradiates the target rear surface in advance with an intensity $\sim 10^{13} \mathrm{~W} / \mathrm{cm}^{2}$ to create the rear plasma with a scale length of $60 \mu \mathrm{m}$. The electron energy spectrum is observed along the UIL axis with an energy spectrometer (ESM) [6] as shown in Fig. 1. The angular distributions of hot electrons are measured with an imaging plate (IP). Hot electrons can enter the ESM after they pass through a hole diameter of $10 \mathrm{~mm}$ made on the IP. The number $N$ of electrons is proportional to the signal intensity $I$ observed with IP. Therefore, the total number $N_{\text {tot }}$ of hot electrons is determined with the integrated signal intensity $I_{\mathrm{IP}}$ on IP from the relation of $N_{\text {tot }}=I_{\mathrm{IP}} N_{\mathrm{L}} / I_{\mathrm{L}}$. Here, the coefficient $N_{\mathrm{L}} / I_{\mathrm{L}}$ is given from the number $N_{\mathrm{L}}$ of electrons on the laser axis measured with the ESM and 

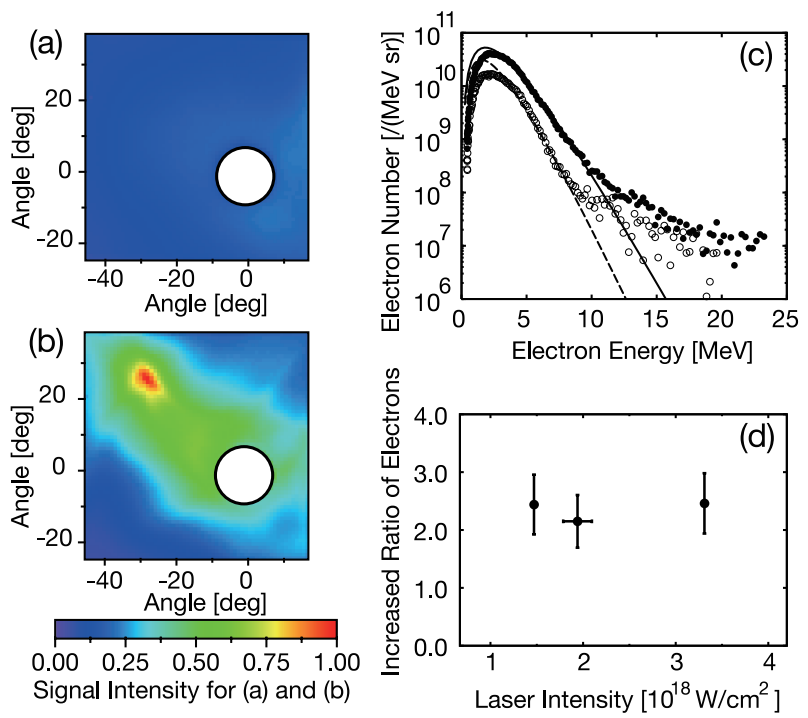

Fig. 2 Hot electron angular distributions observed (a) without and (b) with the rear plasma. (c) Energy spectra observed without the rear plasma (open circle and dashed curve) and with the rear plasma (solid circle and solid curve). (d) The increased ratio of hot electrons for the several UIL intensities.

the signal intensity $I_{\mathrm{L}}$ at the laser axis, which can be estimated from the interpolation of the angular distributions in the hole. The angular distributions and the energy spectra are shown in Figs. 2 (a)-(c) at the UIL intensity $2 \times 10^{18}$ $\mathrm{W} / \mathrm{cm}^{2}$. The distributions shown in Figs. 2 (a) and (b) correspond to the cases without and with the rear plasma. Here, both distributions are normalized by the maximum signal intensity in the rear plasma case. Energy spectra are shown for both cases in Fig. 2 (c). Both electron temperatures are $0.8 \mathrm{MeV}$ estimated by the approximation with a relativistic Maxwellian distribution. The number of hot electrons increases with the rear plasma $\sim 10^{11}$ compared to the without rear plasma case. The ratios of number increase are about 2 for the three different UIL intensities as shown in Fig. 2 (d).

A 1D PIC simulation is employed to investigate the influence of the rear plasma on the electrostatic potential evolution. The simulation is performed with almost actual experimental conditions as shown in Fig. 3(a). The UIL pulse duration and the peak intensity are $700 \mathrm{fs}$ (Gaussian profile) and $5 \times 10^{18} \mathrm{~W} / \mathrm{cm}^{2}$, respectively. The dashed (solid) curve corresponds to the without (with) rear plasma case in the figure. The potential wall that is an energy threshold for electron escape is calculated for an electron departing from the target rear surface at a certain timing as shown in Fig. 3(b). The figure shows that an electron with energy of $2.5 \mathrm{MeV}$, which is the average energy in the experiments, can escape until $\sim 860$ fs in the without rear plasma case. Since the potential evolution is retarded in the rear plasma case, the gate opening time for electron emission is elongated 100-150 fs for the electrons with energies
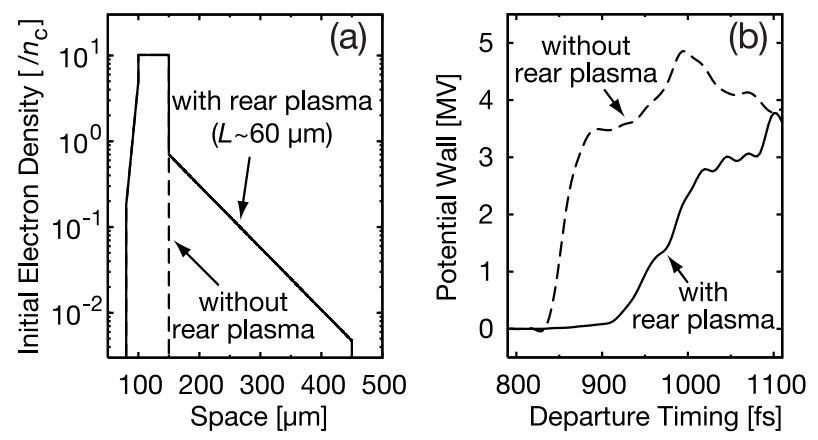

Fig. 3 (a) The initial density profile in the PIC simulation. (b) The potential wall for the electron departing from the target rear surface at a certain timing.

up to $4 \mathrm{MeV}$. The retardation may be closely related to the return current supply in the rear plasma because the electrostatic field production could be neutralized by the return current. The maximum duration $\Delta t$ of the return current supply depends on the electron areal density $\sigma_{\mathrm{r}}$ of the rear plasma as $\int_{0}^{\Delta t} j_{\mathrm{r}} \mathrm{d} t=e \sigma_{\mathrm{r}}$ assuming the 1-dimensional current flow. Here, $j_{\mathrm{r}}$ and $e$ are the density of return current and the electron charge, respectively. The $\sigma_{\mathrm{r}}$ is equal to $n_{\mathrm{p}} L$ for the plasma with the maximum density $n_{\mathrm{p}}$ and the scale length $L$. We obtain the relation: $n_{\mathrm{h}} v_{\mathrm{h}} \Delta t=n_{\mathrm{p}} L$, when the $j_{\mathrm{r}}$ balances with the $j_{\mathrm{h}}\left(j_{\mathrm{h}}=e n_{\mathrm{h}} v_{\mathrm{h}}\right)$. In our arguments, the hot electron density $n_{\mathrm{h}}$ and velocity $v_{\mathrm{h}}$ are assumed to be the critical density $n_{\mathrm{c}}$ for the UIL pulse and the speed of light $c$, respectively. Then the retardation time $\Delta t$ is estimated to be about $140 \mathrm{fs}$ for the rear plasma $\left(n_{\mathrm{p}}=0.7 n_{\mathrm{c}}\right.$ and $L=60 \mu \mathrm{m})$. This estimation is consistent with the simulation results shown in Fig. 3 (b).

If the net current of hot electrons is larger than the Alfvén current as reported in Ref. [7], the magnetic fields produced around the current prevent to flight straight in vacuum. The number of electrons limited by the Alfvén current is calculated as $10^{11}$ within the estimated retardation time (140 fs) for $2.5 \mathrm{MeV}$ electrons (average energy in the experiments). This number agrees well with the observed increase in the experiments. On the other hand, when the hot electrons are produced at the focal spot on the target front, the number produced by the UIL pulse per time is expressed as $\pi n_{\mathrm{h}} v_{\mathrm{h}} r^{2}$, where $r$ is the radius of the hot electron beam resulting in $10^{13}$, much larger than the observed increase. These results strongly imply that the hot electrons are influenced by the magnetic fields as well as the electrostatic potential.

In summary, the emission of hot electrons is studied from the target irradiated by the UIL pulse. The increase in the number of hot electrons is observed with the rear plasma by a factor of 2 at an UIL intensity $\sim 10^{18} \mathrm{~W} / \mathrm{cm}^{2}$. The 1D PIC simulation shows the retardation of the potential evolution, consistent with our model considering the balance of the return and the hot electron currents. The retardation elongates the gate opening time resulting in the 
increase in the number of the emitted hot electrons. The number of electrons is influenced by the Alfvén limit even in the elongated time window when the current produced by the UIL pulse is larger than the Alfvén current.

The authors are very grateful to the staffs for the support in laser operation and the target fabrication in ILE. This work is supported by MEXT, Grant-in Aid for Creative Scientific Research (15GS0214). The part of this research is supported by the CUP on plasma and nuclear fusion operated by NIFS and founded by JSPS. T. M. and A. L. L. acknowledge the support by JSPS.
[1] M. Tabak et al., Phys. Plasmas 1, 1626 (1994).

[2] K. Yasuike et al., Rev. Sci. Instrum. 72, 1236 (2001).

[3] G. Malka et al., Phys. Rev. E 66, 066402 (2002).

[4] A.J. Mackinnon et al., Phys. Rev. Lett. 86, 1769 (2001).

[5] H. Alfvén, Phys. Rev. 55, 425 (1939).

[6] K.A. Tanaka et al., Rev. Sci. Instrum. 76, 013507 (2005).

[7] Y. Sentoku et al., Phys. Rev. E 65, 046408 (2002). 